\title{
On the spectrum of the operator which is a composition of integration and substitution
}

\author{
by \\ Ignat Domanov (Donetsk and Praha)
}

\begin{abstract}
Let $\phi:[0,1] \rightarrow[0,1]$ be a nondecreasing continuous function such that $\phi(x)>x$ for all $x \in(0,1)$. Let the operator $V_{\phi}: f(x) \mapsto \int_{0}^{\phi(x)} f(t) d t$ be defined on $L_{2}[0,1]$. We prove that $V_{\phi}$ has a finite number of nonzero eigenvalues if and only if $\phi(0)>0$ and $\phi(1-\varepsilon)=1$ for some $0<\varepsilon<1$. Also, we show that the spectral trace of the operator $V_{\phi}$ always equals 1 .
\end{abstract}

1. Introduction. It is well known that the Volterra integration operator $V: f(x) \mapsto \int_{0}^{x} f(t) d t$ defined on $L_{2}[0,1]$ is quasinilpotent, i.e., $\sigma(V)=\{0\}$. Let $\phi \in C[0,1]$ be such that $\phi(0)=0$. It was pointed out in [9] and [10] that the operator $V_{\phi}$ defined by

$$
V_{\phi}: f(x) \mapsto \int_{0}^{\phi(x)} f(t) d t
$$

is quasinilpotent on $C[0,1]$ whenever $\phi(x) \leq x$ for all $x \in[0,1]$.

Let $\phi:[0,1] \rightarrow[0,1]$ be a measurable function and let $V_{\phi}: L_{p}[0,1] \rightarrow$ $L_{p}[0,1](1 \leq p<\infty)$ be defined by (1.1). It was proved in [11] and [13] that $V_{\phi}$ is quasinilpotent on $L_{p}[0,1]$ if and only if $\phi(x) \leq x$ for almost all $x \in[0,1]$. It was noted in [13] and proved in [15] that the spectral radius of $V_{x^{\alpha}}$ (defined on $L_{p}[0,1]$ or $\left.C[0,1]\right)$ is $1-\alpha(0<\alpha<1)$. The detailed investigation of the spectrum of the operator $V_{x^{\alpha}}$ was done in [1], where it was shown that the point spectrum $\sigma_{\mathrm{p}}\left(V_{x^{\alpha}}\right)$ of $V_{x^{\alpha}}$ is simple and $\sigma_{\mathrm{p}}\left(V_{x^{\alpha}}\right)=\left\{(1-\alpha) \alpha^{n-1}\right\}_{n=1}^{\infty}$. The oscillation properties of the eigenfunctions of $V_{x^{\alpha}}$ were also investigated in $[1]$.

The aim of this paper is to prove the following theorem.

2000 Mathematics Subject Classification: 34120, 45C05, 47A10, 47A75.

Key words and phrases: eigenvalue, integral operator, Fredholm determinant.

This research was partially supported by NAS of Ukraine, Grant \# 0105 U006289. 
THEOREM 1.1. Let $\phi:[0,1] \rightarrow[0,1]$ be a nondecreasing continuous function such that $\phi(x)>x$ for all $x \in(0,1)$, and $V_{\phi}$ be defined on $L_{2}[0,1]$ by (1.1). Set also $\sigma_{\mathrm{p}}\left(V_{\phi}\right) \backslash\{0\}=\left\{\lambda_{n}\right\}_{n=1}^{\omega}(1 \leq \omega \leq \infty)$. Then:

(1) $\omega<\infty$ if and only if $\phi(0)>0$ and $\phi(1-\varepsilon)=1$ for some $0<\varepsilon<1$;

(2) $\lim _{\varepsilon \rightarrow 0} \sum_{\left|\lambda_{n}\right|>\varepsilon} \lambda_{n}=1$;

(3) $\sum_{n=1}^{\omega}\left|\lambda_{n}\right|^{1+\varepsilon}<\infty$ for all $\varepsilon>0$.

The paper is organized as follows.

In Section 2 we recall some classical results on trace class operators, Fredholm determinants and entire functions. In Section 3 we calculate the Fredholm determinant $D_{V_{\phi}}(\lambda)$ of the operator $V_{\phi}$. In Section 4 we estimate the order of growth of $D_{V_{\phi}}(\lambda)$ and prove Theorem 1.1. It turns out that the matrix trace of $V_{\phi}$ is not defined, but the spectral trace of $V_{\phi}$ does not depend on $\phi$ and always equals 1 . This contrasts with the fact that $\sigma_{\mathrm{p}}\left(V_{x}\right)=\emptyset$. We also find the spectral (= matrix) traces of the $V_{\phi}^{2}$ and $V_{\phi}^{3}$. In Section 5 we assume that $\phi:[0,1] \rightarrow[0,1]$ is a strictly increasing continuous function such that $\operatorname{card}\{x: \phi(x)=x\}<\infty$ and describe the spectrum of $V_{\phi}$. Then we consider $V_{\phi}$ defined on the space $L_{p}[0,1]$.

2. Preliminaries. Here we recall some facts about trace class operators, Fredholm determinants and entire functions.

2.1. Let $K$ be a compact operator defined on an infinite-dimensional Hilbert space $\mathfrak{H}$. Let $s_{n}(K)(n \geq 1)$ be the eigenvalues of $K K^{*}$. The operator $K$ is said to be of class $\mathbf{S}_{p}$ if $\sum_{n=1}^{\infty} s_{n}(K)^{p}<\infty$. The trace $\operatorname{tr} K$ of an operator $K \in \mathbf{S}_{1}$ is defined as its matrix trace: $\operatorname{tr} K=\sum_{n=1}^{\infty}\left(K e_{n}, e_{n}\right)$, where $\left\{e_{n}\right\}_{n=1}^{\infty}$ is some orthonormal basis. It is known that $\operatorname{tr} K$ does not depend on the choice of $\left\{e_{n}\right\}_{n=1}^{\infty}$ and the series $\sum_{n=1}^{\infty}\left(K e_{n}, e_{n}\right)$ converges absolutely. The celebrated theorem of Lidskil (see [4]) says that the matrix trace of an operator $K \in \mathbf{S}_{1}$ is equal to its spectral trace, which is defined as the sum of the eigenvalues of $K$ (counted with algebraic multiplicity):

$$
\operatorname{tr} K=\sum_{n=1}^{\infty}\left(K e_{n}, e_{n}\right)=\sum_{n=1}^{\omega} \lambda_{n}, \quad \omega \leq \infty .
$$

Let $K$ be an integral operator, $(K f)(x)=\int_{0}^{1} k(x, t) f(t) d t$ on $L_{2}[0,1]$. It is well known (see [4]) that if $k(x, t)$ is a continuous function on $[0,1] \times[0,1]$, then $K \in \mathbf{S}_{1}$ and $\operatorname{tr} K$ is given by the integral over the diagonal:

$$
\operatorname{tr} K=\int_{0}^{1} k(t, t) d t .
$$


2.2. Now let $k(x, t)$ be a bounded function on $[0,1] \times[0,1]$. Define

$$
D_{K}(\lambda):=\sum_{n=0}^{\infty} \frac{(-1)^{n}}{n !} A_{n} \lambda^{n}
$$

where $A_{0}:=1$ and

$$
\begin{aligned}
A_{n} & :=\int_{0}^{1} \ldots \int_{0}^{1} K\left(t_{1}, \ldots, t_{n}\right) d t_{1} \ldots d t_{n}, \\
K\left(t_{1}, \ldots, t_{n}\right) & :=\operatorname{det}\left(\begin{array}{ccc}
k\left(t_{1}, t_{1}\right) & \ldots & k\left(t_{1}, t_{n}\right) \\
\vdots & \vdots & \vdots \\
k\left(t_{n}, t_{1}\right) & \ldots & k\left(t_{n}, t_{n}\right)
\end{array}\right)
\end{aligned}
$$

for $n \geq 1$. The function $D_{K}(\lambda)$ is called the Fredholm determinant of $K$. Recall (see $[6,8,12])$ that$$
A_{n}=n ! \iint_{0}^{1} \int_{t_{1}}^{1} \ldots \int_{t_{n-1}}^{1} K\left(t_{1}, \ldots, t_{n}\right) d t_{n} \ldots d t_{1}, \quad n \geq 1 .
$$

Moreover, $D_{K}(\lambda)$ is an entire function of $\lambda$ of order $\varrho \leq 2$, and $D_{K}\left(\mu^{*}\right)=0$ if and only if $\lambda^{*}:=1 / \mu^{*} \in \sigma_{\mathrm{p}}(K)$; moreover, the multiplicity of $\mu^{*}$ as a root of the Fredholm determinant of $K$ is equal to the algebraic multiplicity of the eigenvalue $\lambda^{*}$.

2.3. From Hadamard's theorem ([7, Th. 1, p. 26]) and Lindelöf's theorem ([7, Th. 3, p. 33]), we get the following

THEOREM 2.1. Let $f(z)$ be an entire function of order $\varrho_{f} \leq 1$ and type $\sigma_{f}<\infty$. Let also $\left\{a_{n}\right\}_{n=1}^{\omega}(\omega \leq \infty)$ be all roots of $f(z)$ and $f(0)=1$. Then

(i) if $\varrho_{f}=1, \sigma_{f}=0$ and $\sum_{n=1}^{\omega} 1 /\left|a_{n}\right|<\infty$, then $\omega=\infty, f(z)=$ $\prod_{n=1}^{\infty}\left(1-z / a_{n}\right)$ and $\sum_{n=1}^{\infty} 1 / a_{n}=-f^{\prime}(0)$

(ii) if $\varrho_{f}<1$, then $f(z)=\prod_{n=1}^{\omega}\left(1-z / a_{n}\right)$ and $\sum_{n=1}^{\omega} 1 / a_{n}=-f^{\prime}(0)$;

(iii) if $\varrho_{f}=0$, then $\sum_{n=1}^{\omega} 1 /\left|a_{n}\right|^{\varepsilon}<\infty$ for each $\varepsilon>0$;

(iv) if $\varrho_{f}=1, \sigma_{f}=0$ and $\sum_{n=1}^{\infty} 1 /\left|a_{n}\right|=\infty$, then

$$
f(z)=e^{a z} \prod_{n=1}^{\infty}\left(1-\frac{z}{a_{n}}\right) e^{z / a_{n}} \quad \text { and } \quad \limsup _{r \rightarrow \infty}\left|a+\sum_{\left|a_{n}\right|<r} \frac{1}{a_{n}}\right|=0 .
$$

In particular,

$$
\limsup _{r \rightarrow \infty}\left(\sum_{\left|a_{n}\right|<r} \frac{1}{a_{n}}\right)=-a=-f^{\prime}(0) .
$$

(v) $\sum_{n=1}^{\omega} 1 /\left|a_{n}\right|^{1+\varepsilon}<\infty$ for each $\varepsilon>0$. 
3. The Fredholm determinant of the operator $V_{\phi}$. We begin with an auxiliary lemma.

Lemma 3.1. Let $A=\left(a_{i j}\right)_{i, j=1}^{n}$ be an $n \times n$ matrix all of whose elements are 0 or 1 and $a_{i j}=1$ for $1 \leq j \leq i \leq n$. Then

$$
\operatorname{det} A=\prod_{i=2}^{n}\left(1-a_{i-1, i}\right)= \begin{cases}1, & a_{i-1, i}=0 \text { for } 2 \leq i \leq n, \\ 0, & \text { otherwise }\end{cases}
$$

Proof. The proof is trivial.

TheOREM 3.2. Let $\phi:[0,1] \rightarrow[0,1]$ be a nondecreasing continuous function such that $\phi(x)>x$ for all $x \in(0,1)$. Let $V_{\phi}$ be defined on $L_{2}[0,1]$ by (1.1). Then

$$
D_{V_{\phi}}(\lambda)=1+\sum_{n=1}^{\infty}(-1)^{n} \lambda^{n} \int_{0}^{1} \int_{\phi\left(t_{1}\right)}^{1} \ldots \int_{\phi\left(t_{n-1}\right)}^{1} d t_{n} \ldots d t_{1} .
$$

Proof. It is clear that $\left(V_{\phi} f\right)(x)=\int_{0}^{1} k(x, t) f(t) d t=:(K f)(x)$, where

$$
k(x, t)=\chi(\phi(x)-t)= \begin{cases}1, & \phi(x) \geq t \\ 0, & \phi(x)<t .\end{cases}
$$

Assume that $0 \leq t_{1} \leq \cdots \leq t_{n} \leq 1$. Then $k\left(t_{i}, t_{j}\right)=1$ for $1 \leq j \leq i \leq n$ and the matrix $\left(k\left(t_{i}, t_{j}\right)\right)_{i, j=1}^{n}$ satisfies the assumptions of Lemma 3.1. Hence, $K\left(t_{1}, \ldots, t_{n}\right)=\prod_{i=2}^{n}\left(1-k\left(t_{i-1}, t_{i}\right)\right)$. Further, using $(2.3)-(2.5)$ we get

$$
A_{n}=n ! \iint_{0}^{1} \int_{t_{1}}^{1} \ldots \int_{t_{2}}^{1} \prod_{t_{n-1}}^{n}\left(1-k\left(t_{i-1}, t_{i}\right)\right) d t_{n} \ldots d t_{1}=n ! \int_{\Omega_{n}} 1 d t_{n} \ldots d t_{1},
$$

where

$$
\begin{aligned}
\Omega_{n} & :=\left\{\left(t_{1}, \ldots, t_{n}\right): 0 \leq t_{1} \leq \cdots \leq t_{n} \leq 1, k\left(t_{1}, t_{2}\right)=\cdots=k\left(t_{n-1}, t_{n}\right)=0\right\} \\
& =\left\{\left(t_{1}, \ldots, t_{n}\right): 0 \leq t_{1} \leq \phi\left(t_{1}\right) \leq t_{2} \leq \phi\left(t_{2}\right) \leq \cdots \leq \phi\left(t_{n-1}\right) \leq t_{n} \leq 1\right\} .
\end{aligned}
$$

That is,

$$
A_{n}=n ! \int_{0}^{1} \int_{\phi\left(t_{1}\right)}^{1} \ldots \int_{\phi\left(t_{n-1}\right)}^{1} d t_{n} \ldots d t_{1}, \quad n \geq 1
$$

This completes the proof.

4. The spectrum of the operator $V_{\phi}$. The following proposition immediately follows from Theorem 3.2.

Proposition 4.1. Let $\phi:[0,1] \rightarrow[0,1]$ be a nondecreasing continuous function such that $\phi(x)>x$ for all $x \in(0,1)$. Then $\sigma_{\mathrm{p}}\left(V_{\phi}\right) \cap \mathbb{R}_{-}=\emptyset$. 
Lemma 4.2. Suppose $\phi:[0,1] \rightarrow[0,1]$ is a nondecreasing continuous function and $\phi(x)>x$ for $x \in(0,1)$. Then the following conditions are equivalent:

(i) $\phi(0)>0$ and $\phi(1-\varepsilon)=1$ for some $0<\varepsilon<1$;

(ii) there exists a unique $N=N(\phi) \in\{2,3, \ldots\}$ such that $\phi^{N}(x):=$ $\phi(\phi(\ldots \phi(x)))=1$ for all $x \in[0,1]$ and $\phi^{N-1}\left(x_{0}\right) \neq 1$ for some $x_{0} \in[0,1)$.

Proof. The proof is left to the reader.

TheOREM 4.3. Let $\phi:[0,1] \rightarrow[0,1]$ be a nondecreasing continuous function such that $\phi(x)>x$ for all $x \in(0,1)$. Suppose also that $\phi(0)>0$, $\phi(1-\varepsilon)=1$ for some $0<\varepsilon<1$, and $N=N(\phi)$ is determined by Lemma 4.2(ii). Then

(1) $\sigma_{\mathrm{p}}\left(V_{\phi}\right)=\{0\} \cup\left\{\lambda_{1}, \ldots, \lambda_{N}\right\}$, with all $\lambda_{n} \neq 0$;

(2) $\sum_{n=1}^{N} \lambda_{n}=1$.

Proof. It is easily shown that $0 \in \sigma_{\mathrm{p}}\left(V_{\phi}\right)$. Using Theorem 3.2, we get

$$
D_{V_{\phi}}(\lambda)=1+\sum_{n=1}^{\infty} A_{n} \lambda^{n}, \quad \text { where } \quad A_{n}=(-1)^{n} \int_{0}^{1} \int_{\phi\left(t_{1}\right)}^{1} \ldots \int_{\phi\left(t_{n-1}\right)}^{1} d t_{n} \ldots d t_{1} .
$$

It is easily shown that $\phi^{n-1}\left(t_{1}\right) \leq t_{n} \leq 1$. Since $\phi^{n}(x)=1$ for $n \geq N$, it follows that $A_{n}=0$ for $n \geq N+1$. Therefore $D_{V_{\phi}}(\lambda)$ is a polynomial of degree $N$ and (1) is proved. Further note that $D_{V_{\phi}}(\lambda)=\prod_{n=1}^{N}\left(1-\lambda / a_{n}\right)$. Thus

$$
\sum_{n=1}^{N} \lambda_{n}=\sum_{n=1}^{N} \frac{1}{a_{n}}=-A_{1}=1 .
$$

Let $\alpha_{i}, \beta_{i} \in C[0,1](1 \leq i \leq n)$. Define

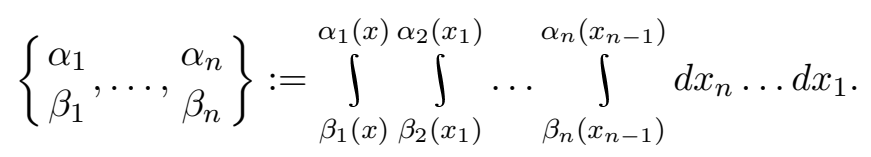

So $\left\{\begin{array}{l}\alpha_{1} \\ \beta_{1}\end{array}, \ldots, \begin{array}{c}\alpha_{n} \\ \beta_{n}\end{array}\right\}$ is a function of $x$. It is clear that

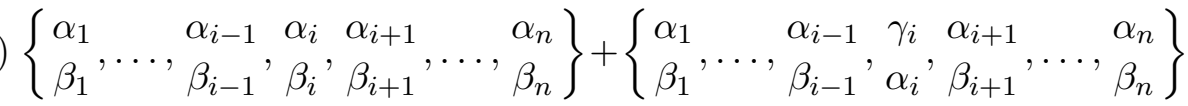

$$
\begin{aligned}
& =\left\{\begin{array}{ccc}
\alpha_{1} \\
\beta_{1}, \ldots, & \alpha_{i-1}, \alpha_{i} \\
\beta_{i-1} & \beta_{i}
\end{array}+\begin{array}{ccc}
\gamma_{i} & \alpha_{i+1} \\
\alpha_{i} & \beta_{i+1}
\end{array}, \ldots, \begin{array}{c}
\alpha_{n} \\
\beta_{n}
\end{array}\right\}
\end{aligned}
$$

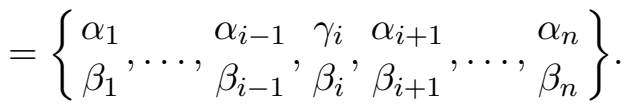

The following lemmas are needed. 
LemmA 4.4. Let $0<\varepsilon_{1}<\varepsilon_{2}<1$ and

$$
\psi(x)= \begin{cases}\psi_{1}(x), & x \in\left[0, \varepsilon_{1}\right], \\ \psi_{2}(x), & x \in\left[\varepsilon_{1}, \varepsilon_{2}\right], \\ \psi_{3}(x), & x \in\left[\varepsilon_{2}, 1\right],\end{cases}
$$

be a strictly increasing continuous function such that $\psi\left(\varepsilon_{1}\right)=\varepsilon_{1}$ and $\psi\left(\varepsilon_{2}\right)$ $=\varepsilon_{2}$. Let also $a_{0}=b_{0}=c_{0}=1$ and $a_{k}, b_{k}, c_{k}, d_{k}(k=1,2, \ldots)$ be the $k$-fold integrals defined by

$$
\begin{aligned}
& a_{k}:=\left\{\begin{array}{ccc}
\varepsilon_{1} & \psi_{1} \\
0 & 0
\end{array}, \ldots, \begin{array}{c}
\psi_{1} \\
0
\end{array}\right\}, \quad b_{k}:=\left\{\begin{array}{cc}
\varepsilon_{2}, & \psi_{2}, \ldots, \\
\varepsilon_{1}, & \varepsilon_{1}
\end{array}, \begin{array}{l}
\varepsilon_{2} \\
\varepsilon_{1}
\end{array}\right\},
\end{aligned}
$$

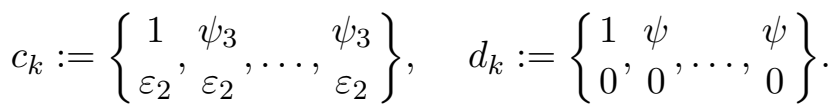

Then

$$
d_{n}=\sum_{k=0}^{n} c_{k} \sum_{l=0}^{n-k} b_{l} a_{n-k-l}, \quad n=1,2, \ldots
$$

Proof. Using (4.1), we get

$$
\begin{aligned}
& d_{n}=\left\{\begin{array}{c}
\varepsilon_{1} \\
0
\end{array}+\begin{array}{l}
\varepsilon_{2} \\
\varepsilon_{1}
\end{array}+\begin{array}{c}
1 \\
\varepsilon_{2}
\end{array}, \begin{array}{l}
\psi \\
0
\end{array}, \ldots, \begin{array}{l}
\psi \\
0
\end{array}\right\}
\end{aligned}
$$

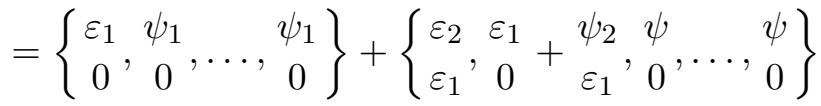

$$
\begin{aligned}
& +\left\{\begin{array}{cc}
1 & \varepsilon_{1} \\
\varepsilon_{2}, & 0
\end{array}+{ }_{\varepsilon_{1}}^{\varepsilon_{2}}+\begin{array}{lll}
\psi_{3} & \psi \\
\varepsilon_{2} & 0 & 0
\end{array}, \begin{array}{l}
\psi \\
0
\end{array}\right\} \\
& =: K_{n}+L_{n}+M_{n} \text {. }
\end{aligned}
$$

By definition $K_{n}=a_{n}$. Further, again using (4.1), we get

$$
\begin{aligned}
& L_{n}=\left\{\begin{array}{cccc}
\varepsilon_{2}, \varepsilon_{1} & \psi_{1} \\
\varepsilon_{1}, & 0 & 0
\end{array}, \ldots, \begin{array}{c}
\psi_{1} \\
0
\end{array}\right\}+\left\{\begin{array}{cccc}
\varepsilon_{2}, & \psi_{2} & \varepsilon_{1} \\
\varepsilon_{1}, & \varepsilon_{1}, & 0
\end{array}+\begin{array}{ccc}
\psi_{2}, & \psi \\
\varepsilon_{1}, & 0
\end{array}, \ldots, 0\right\} \\
& =b_{1} a_{n-1}+\left\{\begin{array}{ccccc}
\varepsilon_{2} & \psi_{2} & \varepsilon_{1} & \psi_{1} & \\
\varepsilon_{1}, & \varepsilon_{1}, & 0 & 0 & \psi_{1} \\
\varepsilon_{1} &
\end{array}\right\}
\end{aligned}
$$

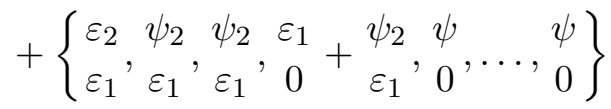

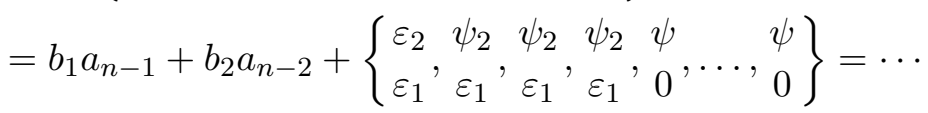

$$
\begin{aligned}
& =\sum_{k=1}^{n} b_{k} a_{n-k} \text {, }
\end{aligned}
$$




$$
\begin{aligned}
& M_{n}=\left\{\begin{array}{cccc}
1 & \varepsilon_{1} & \psi_{1} \\
\varepsilon_{2}, & 0 & 0 & 0
\end{array}, \begin{array}{c}
\psi_{1} \\
0
\end{array}\right\}+\left\{\begin{array}{cccc}
1 & \varepsilon_{2} & \psi_{2} & \psi \\
\varepsilon_{2}, & \varepsilon_{1}, & 0 & 0
\end{array}, \ldots, \begin{array}{l}
\psi \\
0
\end{array}\right\} \\
& +\left\{\begin{array}{ccc}
1 & \psi_{3}, & \psi \\
\varepsilon_{2}, & \varepsilon_{2} & 0
\end{array}, \ldots, \begin{array}{l}
\psi \\
0
\end{array}\right\}
\end{aligned}
$$

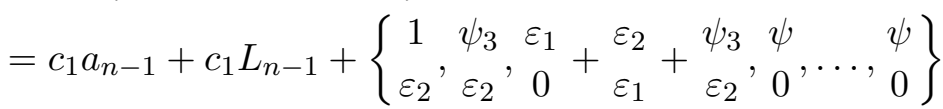

$$
\begin{aligned}
& =c_{1} a_{n-1}+c_{1} L_{n-1}+c_{2} a_{n-2}+c_{2} L_{n-2}
\end{aligned}
$$

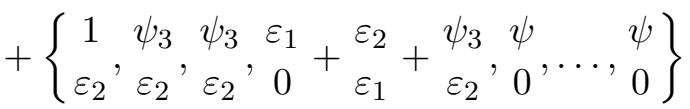

$$
\begin{aligned}
& =\cdots=\sum_{k=1}^{n} c_{k} a_{n-k}+\sum_{k=1}^{n-1} c_{k} L_{n-k} \\
& =\sum_{k=1}^{n} c_{k} a_{n-k}+\sum_{k=1}^{n} c_{k} \sum_{l=1}^{n-k} b_{l} a_{n-k-l} .
\end{aligned}
$$

Finally, we obtain

$$
\begin{aligned}
d_{n} & =K_{n}+L_{n}+M_{n} \\
& =c_{0} a_{n}+\sum_{k=1}^{n} b_{k} a_{n-k}+\sum_{k=1}^{n} c_{k} a_{n-k}+\sum_{k=1}^{n} c_{k} \sum_{l=1}^{n-k} b_{l} a_{n-k-l} \\
& =\sum_{k=0}^{n} c_{k} \sum_{l=0}^{n-k} b_{l} a_{n-k-l} .
\end{aligned}
$$

Lemma 4.5. Let $0<\varepsilon \leq 1 / 4, \beta>1$, and

$$
\psi_{\varepsilon, \beta}(x)= \begin{cases}x, & x \in[0, \varepsilon], \\ \varepsilon+(1-2 \varepsilon)^{1-\beta}(x-\varepsilon)^{\beta}, & x \in[\varepsilon, 1-\varepsilon], \\ x, & x \in[1-\varepsilon, 1] .\end{cases}
$$

Then

$$
\begin{aligned}
d_{n}:= & \left\{\begin{array}{cc}
1 & \psi_{\varepsilon, \beta} \\
0 & 0
\end{array}, \begin{array}{c}
\psi_{\varepsilon, \beta} \\
0
\end{array}\right\} \\
= & \frac{(2 \varepsilon)^{n}}{n !}+\frac{(1-2 \varepsilon)(2 \varepsilon)^{n-1}}{(n-1) !} \\
& +\sum_{l=2}^{n} \frac{(1-2 \varepsilon)^{l}(2 \varepsilon)^{n-l}}{(n-l) !(1+\beta) \ldots\left(1+\beta+\ldots \beta^{l-1}\right)}, \quad n=1,2, \ldots
\end{aligned}
$$

Moreover,

$$
d_{n}<\operatorname{const}(\varepsilon, \beta) \frac{(4 \varepsilon)^{n}}{n !}, \quad n=1,2, \ldots,
$$

where $\operatorname{const}(\varepsilon, \beta)$ does not depend on $n$. 
Proof. Substituting $\psi_{\varepsilon, \beta}$ for $\psi(x)$ in Lemma 4.4, we get (4.2). Indeed, it is easily proved that $a_{l}=c_{l}=\varepsilon^{l} / l !(l=0,1, \ldots n)$. Define $\widetilde{b}_{1}(x):=$ $(1-2 \varepsilon)^{1-\beta}(x-\varepsilon)^{\beta}, \psi_{2}(x):=\varepsilon+\widetilde{b}_{1}(x)$, and

$$
\widetilde{b}_{l}(x):=\left\{\begin{array}{c}
\psi_{2}, \ldots, \begin{array}{c}
\psi_{2} \\
\varepsilon, \ldots, \varepsilon
\end{array}
\end{array}\right\}, \quad l=2,3, \ldots
$$

Then $\widetilde{b}_{l+1}(x)=\int_{\varepsilon}^{\psi_{2}(x)} \widetilde{b}_{l}(t) d t$. It can easily be checked (by induction on $l$ ) that

$$
\widetilde{b}_{l}(x)=\frac{(1-2 \varepsilon)^{l-\beta-\cdots-\beta^{l}}(x-\varepsilon)^{\beta+\beta^{2}+\cdots+\beta^{l}}}{(1+\beta) \ldots\left(1+\beta+\cdots+\beta^{l-1}\right)}, \quad l=2,3, \ldots .
$$

Since $b_{l}=\widetilde{b}_{l}(1-\varepsilon)$, we see that

$$
\begin{aligned}
& b_{0}=1, \quad b_{1}=1-2 \varepsilon, \\
& b_{l}=\frac{(1-2 \varepsilon)^{l}}{(1+\beta) \ldots\left(1+\beta+\cdots+\beta^{l-1}\right)}, \quad l=2,3, \ldots .
\end{aligned}
$$

Using Lemma 4.4, we get

$$
\begin{aligned}
d_{n} & =\sum_{k=0}^{n} c_{k} \sum_{l=0}^{n-k} b_{l} a_{n-k-l}=\sum_{l=0}^{n} b_{l} \sum_{k=0}^{n-l} c_{k} a_{n-k-l} \\
& =\sum_{l=0}^{n} b_{l} \sum_{l=0}^{n-l} \frac{\varepsilon^{k}}{k !} \frac{\varepsilon^{n-k-l}}{(n-k-l) !} \\
& =\sum_{l=0}^{n} b_{l} \frac{\varepsilon^{n-l}}{(n-l) !} \sum_{l=0}^{n-l} \frac{(n-l) !}{k !(n-l-k) !} \\
& =\sum_{l=0}^{n} b_{l} \frac{(2 \varepsilon)^{n-l}}{(n-l) !}, \quad n=1,2, \ldots
\end{aligned}
$$

Substituting (4.3) for $b_{l}$ in (4.4) we get (4.2).

To estimate $d_{n}$, taking into account the inequality of arithmetic and geometric means, we obtain

$$
(1+\beta) \cdots\left(1+\beta+\cdots+\beta^{l-1}\right) \geq 2 \beta^{1 / 2} 3 \beta^{2 / 2} \ldots l \beta^{(l-1) / 2}=l ! \beta^{(l-1) l / 4} .
$$

Hence,

$$
b_{l} \leq \frac{(1-2 \varepsilon)^{l}}{l !}\left(\frac{1}{\beta^{1 / 4}}\right)^{l^{2}-l}<\frac{(1-2 \varepsilon)^{l}}{l !} .
$$

Let $N$ be a number such that

$$
\left(\frac{1}{\beta^{1 / 4}}\right)^{l^{2}-l}<\left(\frac{2 \varepsilon}{1-2 \varepsilon}\right)^{l} \quad \text { for } l>N
$$


(for example, $N=\left[4 \log _{\beta}(1 /(2 \varepsilon)-1)\right]+2$ ). Then $b_{l}<(2 \varepsilon)^{l} / l$ ! for $l>N$. Using (4.4), we get, for $n>N$,

$$
\begin{aligned}
d_{n} & =\sum_{l=0}^{N} b_{l} \frac{(2 \varepsilon)^{n-l}}{(n-l) !}+\sum_{l=N+1}^{n} b_{l} \frac{(2 \varepsilon)^{n-l}}{(n-l) !} \\
& \leq \frac{(2 \varepsilon)^{n}}{n !} \sum_{l=0}^{N} \frac{n !}{l !(n-l) !}\left(\frac{1-2 \varepsilon}{2 \varepsilon}\right)^{l}+\frac{(2 \varepsilon)^{n}}{n !} \sum_{l=N+1}^{n} \frac{n !}{l !(n-l) !} \\
& \leq \frac{(2 \varepsilon)^{n}}{n !}\left(\frac{1-2 \varepsilon}{2 \varepsilon}\right)^{N} \sum_{l=0}^{N} \frac{n !}{l !(n-l) !}+\frac{(2 \varepsilon)^{n}}{n !} \sum_{l=0}^{n} \frac{n !}{l !(n-l) !} \\
& \leq \frac{(4 \varepsilon)^{n}}{n !}\left(\left(\frac{1-2 \varepsilon}{2 \varepsilon}\right)^{N}+1\right) .
\end{aligned}
$$

This completes the proof.

LemMa 4.6. Let $\beta>1$ and

$$
\psi_{\beta}(x):= \begin{cases}2^{\beta-1} x^{\beta}=: \psi_{1}(x), & x \in[0,1 / 2], \\ 2^{\beta-1}(x-1 / 2)^{\beta}+1 / 2=: \psi_{2}(x), & x \in[1 / 2,1] .\end{cases}
$$

Let $a_{0}=b_{0}=1$ and $a_{k}, b_{k}$, and $d_{k}(k=1,2 \ldots)$ be the $k$-fold integrals defined by

$$
\begin{aligned}
& a_{k}:=\left\{\begin{array}{ccc}
1 / 2 & \psi_{1} & \psi_{1} \\
0 & 0
\end{array}, \ldots, \quad b_{k}:=\left\{\begin{array}{ccc}
1 & \psi_{2}, \ldots, & \psi_{2} \\
1 / 2 & 1 / 2
\end{array}\right\},\right. \\
& d_{k}:=\left\{\begin{array}{ccc}
1 & \psi_{\beta} \\
0 & 0
\end{array}, \ldots, \begin{array}{c}
\psi_{\beta} \\
0
\end{array}\right\} \text {. }
\end{aligned}
$$

Then

$$
\begin{array}{ll}
d_{n}=\sum_{l=0}^{n} b_{l} a_{n-l}, & n=1,2, \ldots, \\
d_{n}<\frac{\beta^{\left(-n^{2} / 2+n\right) / 4}}{n !}, & n=1,2, \ldots .
\end{array}
$$

Proof. Substituting $1 / 2$ for $\varepsilon_{1}$ and 1 for $\varepsilon_{2}$ in Lemma 4.4, we get (4.6). Further, it is not hard to prove that $a_{1}=b_{1}=1 / 2$ and

$$
a_{l}=b_{l}=2^{-l}\left((\beta+1) \cdots\left(\beta^{l-1}+\cdots+1\right)\right)^{-1} \quad \text { for } l \geq 2 .
$$

Now, by (4.5), $a_{l} \leq 2^{-l} / \beta^{(l-1) l / 4} l$ ! and

$$
\begin{aligned}
d_{n} & \leq \sum_{l=0}^{n} \frac{2^{-l}}{\beta^{(l-1) l / 4} l !} \frac{2^{-n+l}}{\beta^{(n-l-1)(n-l) / 4}(n-l) !} \\
& =\frac{2^{-n}}{n !} \sum_{l=0}^{n} \frac{n !}{l !(n-l) !} \beta^{\left(-2(l-n / 2)^{2}-n^{2} / 2+n\right) / 4}<\frac{\beta^{\left(-n^{2} / 2+n\right) / 4}}{n !} .
\end{aligned}
$$


Proposition 4.7. Let $\phi:[0,1] \rightarrow[0,1]$ be a nondecreasing continuous function.

(1) If $\phi(x)>x$ for $x \in(0,1)$ then the order of $D_{V_{\phi}}(\lambda)$ does not exceed 1 , and if it equals $1, D_{V_{\phi}}(\lambda)$ is of minimal type;

(2) if for some $0<a<b<1$,

$$
\phi(x) \geq f_{a, b}(x):= \begin{cases}\frac{b}{a} x, & x \in[0, a], \\ \frac{1-b}{1-a} x+\frac{b-a}{1-a}, & x \in[a, 1],\end{cases}
$$

for $x \in[0,1]$, then the order of $D_{V_{\phi}}(\lambda)$ equals 0 .

Proof. (1) Taking into account Theorem 3.2, we obtain

$$
D_{V_{\phi}}(\lambda)=1+\sum_{n=1}^{\infty}(-1)^{n} A_{n} \lambda^{n}, \quad \text { where } \quad A_{n}=\left\{\begin{array}{lll}
1 & 1 & 1 \\
0 & \phi
\end{array}, \ldots, \quad, \phi .\right.
$$

Since $\phi(x)>x$ for each $0<\varepsilon<1 / 4$, it follows that there exists $\beta>1$ such that $\phi(x) \geq \psi_{\varepsilon, \beta}^{-1}(x)$. Using Lemma 4.5 , we get

$$
\begin{aligned}
& A_{n}=d_{n}=\left\{\begin{array}{cccc}
1 & 1 & 1 \\
0 & \phi
\end{array}, \ldots, \phi\left\{\begin{array}{ccc}
1 & 1 & 1 \\
0 & \psi_{\varepsilon, \beta}^{-1}, \ldots, & \psi_{\varepsilon, \beta}^{-1}
\end{array}\right\}\right. \\
& =\left\{\begin{array}{ccc}
1 & \psi_{\varepsilon, \beta} \\
0 & 0
\end{array}, \ldots, \begin{array}{c}
\psi_{\varepsilon, \beta} \\
0
\end{array}\right\}<\operatorname{const}(\varepsilon, \beta) \frac{(4 \varepsilon)^{n}}{n !} .
\end{aligned}
$$

Therefore the order of growth of $D_{V_{\phi}}(\lambda)$ does not exceed 1. Assume that this order is 1 . Then the type of $D_{V_{\phi}}(\lambda)$ does not exceed $4 \varepsilon$ for each $\varepsilon<1 / 4$. Thus $D_{V_{\phi}}(\lambda)$ is of minimal type.

(2) Since $\phi(x) \geq f_{a, b}(x)$ for some $0<a<b<1$, it follows that there exists $\beta>1$ such that $\phi(x) \geq \psi_{\beta}^{-1}(x)$. Using Lemma 4.6, we get

$$
\begin{aligned}
A_{n}=d_{n} & =\left\{\begin{array}{lll}
1 & 1 & 1 \\
0 & \phi
\end{array}, \ldots, \begin{array}{l}
\phi
\end{array}\right\}<\left\{\begin{array}{ccc}
1 & 1 & 1 \\
0 & \psi_{\beta}^{-1}, \ldots, & \psi_{\beta}^{-1}
\end{array}\right\} \\
& =\left\{\begin{array}{cc}
1 & \psi_{\beta} \\
0 & 0
\end{array}, \ldots, \begin{array}{c}
\psi_{\beta} \\
0
\end{array}\right\}<\frac{\beta^{\left(-n^{2} / 2+n\right) / 4}}{n !} .
\end{aligned}
$$

Therefore the order of growth of $D_{V_{\phi}}(\lambda)$ equals 0 .

TheOREM 4.8. Let $\phi:[0,1] \rightarrow[0,1]$ be a nondecreasing continuous function such that $\phi(x)>x$ for all $x \in(0,1)$. Suppose that either $\phi(0)=0$ or $\phi(1-\varepsilon) \neq 1$ for all $0<\varepsilon<1$. Then

(1) $\sigma_{\mathrm{p}}\left(V_{\phi}\right) \backslash\{0\}=:\left\{\lambda_{1}, \lambda_{2}, \ldots\right\}$ is an infinite set;

(2) $\lim _{\varepsilon \rightarrow 0} \sum_{\left|\lambda_{n}\right|>\varepsilon} \lambda_{n}=1$;

(3) $\sum_{n=1}^{\omega}\left|\lambda_{n}\right|^{1+\varepsilon}<\infty$ for all $\varepsilon>0$. 
Proof. Using Theorem 3.2, we get

$$
D_{V_{\phi}}(\lambda)=1+\sum_{n=1}^{\infty}(-1)^{n} A_{n} \lambda^{n}, \quad \text { where } \quad A_{n}=\left\{\begin{array}{lll}
1 & 1 & 1 \\
0 & \phi
\end{array}, \ldots, \quad, \phi .\right.
$$

It is easy to see that if either $\phi(0)=0$ or $\phi(1-\varepsilon) \neq 1$ for all $0<\varepsilon<1$, then $A_{n}>0$ for $n \geq 0$. Therefore $D_{V_{\phi}}(\lambda)$ is not a polynomial in $\lambda$. Now we apply Proposition 4.7(1). Suppose that the order of $D_{V_{\phi}}(\lambda)$ is less than 1; then using Theorem 2.1(ii), we get $D_{V_{\phi}}(\lambda)=\prod_{n=1}^{\omega}\left(1-\lambda / a_{n}\right)$. Since $D_{V_{\phi}}(\lambda)$ is not a polynomial, it follows that $\omega=\infty$ and $\sum_{n=1}^{\infty} \lambda_{n}=\sum_{n=1}^{\infty} 1 / a_{n}=$ $-A_{1} / A_{0}=1$. Now suppose that the order of $D_{V_{\phi}}(\lambda)$ is 1 ; then $D_{V_{\phi}}(\lambda)$ is of minimal type. Thus the spectrum of $V_{\phi}$ is an infinite set. Now, the application of Theorem 2.1(i), (iv) yields (2).

(3) follows from Theorem 2.1.

Now we are ready to prove the main result of the paper.

Proof of Theorem 1.1. (1) follows from Theorem 4.3(1) and Theorem 4.8(1).

(2)-(3) follow from Theorem 4.3(2) and Theorem 4.8(2)-(3).

Theorem 4.9. Let $\phi:[0,1] \rightarrow[0,1]$ be a nondecreasing continuous function and for some $0<a<b<1$,

$$
\phi(x) \geq \begin{cases}\frac{b}{a} x, & x \in[0, a], \\ \frac{1-b}{1-a} x+\frac{b-a}{1-a}, & x \in[a, 1],\end{cases}
$$

for all $x \in[0,1]$. Suppose that either $\phi(0)=0$ or $\phi(1-\varepsilon) \neq 1$ for all $0<\varepsilon<1$. Then

(1) $\sigma_{\mathrm{p}}\left(V_{\phi}\right) \backslash\{0\}=:\left(\lambda_{1}, \lambda_{2}, \ldots\right)$ is an infinite set;

(2) $\sum_{n=1}^{\infty} \lambda_{n}=1$;

(3) $\sum_{n=1}^{\infty}\left|\lambda_{n}\right|^{\varepsilon}<\infty$ for all $\varepsilon>0$.

Proof. (1) follows from Theorem 4.8(1). By Proposition 4.7(2), the order of $D_{V_{\phi}}(\lambda)$ equals 0 . Thus (2) and (3) follow from (ii) and (iii) of Theorem 2.1.

REMARK 4.10. (i) Suppose $\phi$ is strictly increasing and $\phi(x)>x$ for all $x \in(0,1)$. Assume that also $\phi \in C^{1}[0,1]$ and $\left(\phi^{\prime}\right)^{-1 / 2} \in L_{\infty}[0,1]$. We claim that $V_{\phi} \notin \mathbf{S}_{1}$. Indeed, let $c:=\left(\int_{0}^{1}\left(\phi^{\prime}(s)\right)^{1 / 2} d s\right)^{-1}$ and let $W_{\phi}$ and $T_{\phi}$ be the linear operators on $L_{2}[0,1]$ defined by

$$
\left(W_{\phi} f\right)(x)=\int_{0}^{x}\left(\phi^{\prime}(t)\right)^{1 / 2} f(t) d t, \quad\left(T_{\phi} f\right)(x)=f\left(c \int_{0}^{x}\left(\phi^{\prime}(s)\right)^{1 / 2} d s\right) .
$$


It can easily be checked (see [2]-[3]) that $T_{\phi}$ and $T_{\phi}^{-1}$ are bounded operators and $c V_{x}=T_{\phi}^{-1} W_{\phi} T_{\phi}$. Hence (see [5]),

$$
s_{n}\left(W_{\phi}\right) \geq\left\|T_{\phi}\right\|^{-1}\left\|T_{\phi}^{-1}\right\|^{-1} s_{n}\left(c V_{x}\right)=\left\|T_{\phi}\right\|^{-1}\left\|T_{\phi}^{-1}\right\|^{-1} c \frac{2}{(2 n-1) \pi} .
$$

Further,

$$
\left(V_{\phi} V_{\phi}^{*} f\right)(x)=\int_{0}^{\phi(x)} \int_{\phi^{-1}(t)}^{1} f(s) d s d t=\int_{0}^{x} \phi^{\prime}(t) \int_{t}^{1} f(s) d s d t=\left(W_{\phi} W_{\phi}^{*} f\right)(x) .
$$

Thus $s_{n}\left(V_{\phi}\right)=s_{n}\left(W_{\phi}\right) \geq\left\|T_{\phi}\right\|^{-1}\left\|T_{\phi}^{-1}\right\|^{-1} c \frac{2}{(2 n-1) \pi}$. Hence, $V_{\phi} \notin \mathbf{S}_{1}$.

(ii) Since $V_{\phi} \notin \mathbf{S}_{1}$, it follows that the matrix trace of the operator $V_{\phi}$ is not defined. Hence we cannot use $(2.1)-(2.2)$ to prove Theorem $4.9(2)$. Nevertheless, (2.1)-(2.2) hold for $K=V_{\phi}$ and the orthonormal basis $\left\{e_{n}\right\}_{n=1}^{\infty}$ defined by: $e_{1} \equiv 1, e_{2 n}:=e^{2 \pi i n x}$ and $e_{2 n+1}:=e^{-2 \pi i n x}(n=1,2, \ldots)$. Indeed, since $\sum_{n=1}^{\infty}(\sin n x) / n=(\pi-x) / 2$ for $x \in(0,2 \pi)$, it follows that

$$
\begin{aligned}
& \sum_{n=0}^{\infty}\left(V_{\phi} e_{n}, e_{n}\right)=\int_{0}^{1} \phi(x) d x \\
& \quad+\sum_{n=1}^{\infty}\left(\int_{0}^{1} \frac{\left(e^{2 \pi i n \phi(x)}-1\right) e^{-2 \pi i n x}}{2 \pi i n} d x+\int_{0}^{1} \frac{\left(e^{-2 \pi i n \phi(x)}-1\right) e^{2 \pi i n x}}{-2 \pi i n} d x\right) \\
& =\int_{0}^{1} \phi(x) d x+\sum_{n=1}^{\infty} \int_{0}^{1} \frac{\sin (2 \pi n(\phi(x)-x))}{\pi n} d x \\
& =\int_{0}^{1} \phi(x) d x+\int_{0}^{1} \frac{1}{\pi} \frac{\pi-2 \pi(\phi(x)-x)}{2} d x=1 .
\end{aligned}
$$

Further, $\int_{0}^{1} \chi(\phi(x)-x) d x=1$. Thus formulas (2.1)-(2.2) hold. This contrasts with the fact that $\sum_{n=0}^{\infty}\left(V_{x} e_{n}, e_{n}\right)=\infty$.

(iii) Theorem 1.1 states that the spectral trace of an operator $V_{\phi}$ always equals 1 . This also contrasts with the fact that the operator $V_{x}$ is quasinilpotent.

To estimate the spectral radius $r\left(V_{\phi}\right)$ we recall (see [14]) some results on integral operators with nonnegative kernels. Let $(K f)(x)=\int_{0}^{1} k(x, t) f(t) d t$ and $k(x, t) \geq 0$ for $(x, t) \in[0,1] \times[0,1]$. If there exist $\alpha>0$ and a nonnegative function $f$ such that $(K f)(x) \geq \alpha f(x)$ for $x \in[0,1]$, then $r(K) \in \sigma_{\mathrm{p}}(K)$ and $r(K)>\alpha$.

Proposition 4.11. Let $\phi:[0,1] \rightarrow[0,1]$ be a strictly increasing continuous function such that $\phi(x) \geq x$ for all $x \in[0,1]$. Write $\sigma_{\mathrm{p}}\left(V_{\phi}\right)=\left\{\lambda_{n}\right\}_{n=1}^{\omega}$ $(\omega \leq \infty)$. Then 
(1) $r\left(V_{\phi}\right) \geq \max _{x \in[0,1]}(\phi(x)-x), r\left(V_{\phi}\right) \in \sigma_{\mathrm{p}}\left(V_{\phi}\right)$.

Suppose moreover that $\phi(0)=0$. Then $\omega=\infty$ and

(2) $\sum_{n=1}^{\infty} \lambda_{n}^{2}=2 \int_{0}^{1} \phi(t) d t-1$

(3) $\sum_{n=1}^{\infty} \lambda_{n}^{3}=1-3 \int_{0}^{1} \phi(t) \phi^{-1}(t) d t$.

Proof. (1) Let $f_{a}(x)=1-\chi(a-x), a \in(0,1)$. Then

$$
\left(V_{\phi} f_{a}\right)(x)=\left\{\begin{array}{ll}
0, & {\left[0, \phi^{-1}(a)\right]} \\
\phi(x)-a, & {\left[\phi^{-1}(a), 1\right]}
\end{array}\right\} \geq(\phi(a)-a) f_{a}(x),
$$

and (1) is proved.

$(2),(3)$ It is easy to check that $\phi^{-1}(x)$ is well defined and

$$
\begin{aligned}
& \left(V_{\phi}^{2} f\right)(x)=\int_{0}^{1} \chi\left(\phi^{2}(x)-t\right)\left(\phi(x)-\phi^{-1}(t)\right) f(t) d t=: \int_{0}^{1} k_{2}(x, t) f(t) d t, \\
& \left(V_{\phi}^{3} f\right)(x)=\int_{0}^{1} \chi\left(\phi^{3}(x)-t\right) \int_{\phi^{-2}(t)}^{\phi(x)}\left(\phi(s)-\phi^{-1}(t)\right) d s f(t) d t=: \int_{0}^{1} k_{3}(x, t) f(t) d t .
\end{aligned}
$$

Further, $k_{2}$ and $k_{3}$ are continuous functions on $[0,1] \times[0,1]$. Hence, $V_{\phi}^{2} \in \mathbf{S}_{1}$ and $V_{\phi}^{3} \in \mathbf{S}_{1}$. Now if we recall (2.2), we get

$$
\begin{aligned}
\sum_{n=1}^{\infty} \lambda_{n}^{2} & =\int_{0}^{1} k_{2}(t, t) d t=\int_{0}^{1}\left(\phi(t)-\phi^{-1}(t)\right) d t=2 \int_{0}^{1} \phi(t) d t-1, \\
\sum_{n=1}^{\infty} \lambda_{n}^{3} & =\int_{0}^{1} k_{3}(t, t) d t=\int_{0}^{1} \int_{\phi^{-2}(t)}^{\phi(t)}\left(\phi(s)-\phi^{-1}(t)\right) d s \\
& =\int_{0}^{1}\left(\phi(t) \phi^{2}(t)-2 \phi^{-1}(t) \phi(t)+\phi^{-1}(t) \phi^{-2}(t)\right) d t \\
& =1-3 \int_{0}^{1} \phi(t) \phi^{-1}(t) d t .
\end{aligned}
$$

EXAMPLE 4.12 . Let $\phi(x)=x^{\alpha}(0<\alpha<1)$. It can be proved by direct calculations that

$$
D_{V_{x^{\alpha}}}(\lambda)=1+\sum_{n=1}^{\infty}(-1)^{n} \lambda^{n} \int_{0}^{1} \int_{t_{1}^{\alpha}}^{1} \ldots \int_{t_{n-1}^{\alpha}}^{1} d t_{n} \ldots d t_{1}
$$




$$
=1+\sum_{n=1}^{\infty}(-1)^{n} \lambda^{n} \frac{\alpha^{n(n-1) / 2}(1-\alpha)^{n}}{(1-\alpha) \ldots\left(1-\alpha^{n}\right)}=\prod_{n=1}^{\infty}\left(1-\frac{\lambda}{(1-\alpha) \alpha^{n-1}}\right) .
$$

Hence, $\sigma_{\mathrm{p}}\left(V_{x^{\alpha}}\right)=\left\{(1-\alpha) \alpha^{n-1}\right\}_{n=1}^{\infty}$ and each eigenvalue of $V_{x^{\alpha}}$ is of algebraic multiplicity one. Further,

$$
\sum_{n=1}^{\infty}(1-\alpha) \alpha^{n-1}=1 \quad \text { and } \quad \sum_{n=1}^{\infty}\left((1-\alpha) \alpha^{n-1}\right)^{\varepsilon}=\frac{(1-\alpha)^{\varepsilon}}{1-\alpha^{\varepsilon}}<\infty
$$

for each $\varepsilon>0$.

\section{Some generalizations}

5.1. The following lemma can be proved by direct calculations.

Lemma 5.1. Let $A$ be a compact operator defined on a Hilbert space $\mathfrak{H}$. Let $\mathfrak{H}=\bigoplus_{i=1}^{k} \mathfrak{H}_{i}$ and $A_{i}:=P_{i} A: \mathfrak{H}_{i} \rightarrow \mathfrak{H}_{i}$, where $P_{i}$ be the orthogonal projection in $\mathfrak{H}$ onto $\mathfrak{H}_{i}$. Suppose that $\left\{\bigoplus_{j=1}^{i} \mathfrak{H}_{j}\right\}_{i=1}^{k}$ is invariant for $A$. Then $1 / \lambda$ is an eigenvalue of $A$ of algebraic multiplicity $m \geq 1$ if and only if $1 / \lambda$ is an eigenvalue of $A_{i}$ of algebraic multiplicity $m_{i} \geq 0$ and $\sum_{i=1}^{k} m_{i}=m$.

Proof. The proof is omitted.

TheOREM 5.2. Let $\phi:[0,1] \rightarrow[0,1]$ be a strictly increasing continuous function. Let $\{x: \phi(x)=x, x \in(0,1)\}=\left\{a_{i}\right\}_{i=1}^{k-1}$, where $0<a_{1}<\cdots<$ $a_{k-1}<1(k \geq 2)$. Define $a_{0}:=0, a_{k}:=1$, and

$$
\begin{aligned}
& \phi_{i}(x):=\left(\phi\left(x\left(a_{i}-a_{i-1}\right)+a_{i-1}\right)-a_{i-1}\right) /\left(a_{i}-a_{i-1}\right), \quad 1 \leq i \leq k .
\end{aligned}
$$

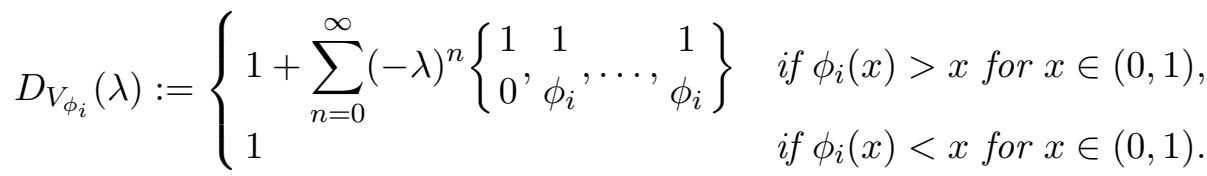

Then

(1) $1 / \lambda \in \sigma_{\mathrm{p}}\left(V_{\phi}\right)$ if and only if $\prod_{i=1}^{k} D_{V_{\phi_{i}}}\left(\left(a_{i}-a_{i-1}\right) \lambda\right)=0$;

(2) the algebraic multiplicity of the eigenvalue $1 / \lambda$ is equal to the multiplicity of $\lambda$ as a root of the entire function $\prod_{i=1}^{k} D_{V_{\phi_{i}}}\left(\left(a_{i}-a_{i-1}\right) \lambda\right)$.

Proof. Set $\mathfrak{H}:=L_{2}[0,1], \mathfrak{H}_{i}:=L_{2}\left[a_{i-1}, a_{i}\right]$ and

$$
\begin{gathered}
P_{i}: f(x) \rightarrow\left\{\begin{array}{ll}
f(x), & x \in\left[a_{i-1}, a_{i}\right], \\
0, & x \notin\left[a_{i-1}, a_{i}\right],
\end{array} \quad P_{i}: \mathfrak{H} \rightarrow \mathfrak{H}_{i},\right. \\
A:=V_{\phi}, \quad A_{i}:=P_{i} A 1_{\mathfrak{H}_{i}}, \\
T_{i}:\left\{\begin{array}{ll}
f(x), & x \in\left[a_{i-1}, a_{i}\right] \\
0, & x \notin\left[a_{i-1}, a_{i}\right]
\end{array}\right\} \mapsto f\left(\left(a_{i}-a_{i-1}\right) x+a_{i-1}\right), \quad T_{i}: \mathfrak{H}_{i} \rightarrow \mathfrak{H} .
\end{gathered}
$$


It follows easily that $\bigoplus_{j=1}^{i} \mathfrak{H}_{j}\left(=L_{2}\left[0, a_{i}\right]\right)$ is invariant for $A$ and

$$
\begin{aligned}
& A_{i}:\left\{\begin{array}{ll}
f(x), & x \in\left[a_{i-1}, a_{i}\right] \\
0, & x \notin\left[a_{i-1}, a_{i}\right]
\end{array}\right\} \mapsto \begin{cases}\int_{a_{n-1}}^{\phi(x)} f(t) d t, & x \in\left[a_{i-1}, a_{i}\right], \\
0, & x \notin\left[a_{i-1}, a_{i}\right],\end{cases} \\
& T_{i}^{-1}: f(x) \mapsto \begin{cases}f\left(\frac{x-a_{i-1}}{a_{i}-a_{i-1}}\right), & x \in\left[a_{i-1}, a_{i}\right], \\
0, & x \notin\left[a_{i-1}, a_{i}\right],\end{cases} \\
& T_{i} A_{i} T_{i}^{-1}=\left(a_{i}-a_{i-1}\right) V_{\phi_{i}} .
\end{aligned}
$$

The application of Theorem 3.2 yields

$$
1 / \lambda \in \sigma_{\mathrm{p}}\left(A_{i}\right) \Leftrightarrow 1 / \lambda \in \sigma_{\mathrm{p}}\left(\left(a_{i}-a_{i-1}\right) V_{\phi_{i}}\right) \Leftrightarrow D_{V_{\phi_{i}}}\left(\left(a_{i}-a_{i-1}\right) \lambda\right)=0 .
$$

Now applying Lemma 5.1 completes the proof.

Corollary 5.3. Suppose $\phi$ satisfies the conditions of Theorem 5.2 and $\operatorname{mes}\{x: \phi(x) \geq x, x \in[0,1]\}>0$. Set also $\sigma_{\mathrm{p}}\left(V_{\phi}\right) \backslash\{0\}=\left\{\lambda_{n}\right\}_{n=1}^{\omega}(1 \leq \omega$ $\leq \infty)$. Then

(1) $\omega<\infty$ if and only if $\phi(0)>0, \phi(1-\varepsilon)=1$ for some $0<\varepsilon<1$ and $\phi(x)>x$ for all $x \in(0,1)$;

(2) $\lim _{\varepsilon \rightarrow 0} \sum_{\left|\lambda_{n}\right|>\varepsilon} \lambda_{n}=\operatorname{mes}\{x: \phi(x) \geq x, x \in[0,1]\}$.

Proof. (1) follows from Theorems 4.3, 4.8, 5.2.

(2) By definition, put

$$
\begin{gathered}
\Omega:=\left\{i: \phi(x) \geq x \text { for } x \in\left[a_{i-1}, a_{i}\right]\right\}=\left\{i: \phi_{i}(x) \geq x \text { for } x \in[0,1]\right\}, \\
\sigma_{\mathrm{p}}\left(V_{\phi_{i}}\right):=\left\{\lambda_{i n}\right\}_{n=1}^{\omega_{i}}, \quad 1 \leq \omega \leq \infty, \quad i \in \Omega .
\end{gathered}
$$

By Theorem 5.2,

$$
\left\{\lambda_{n}\right\}_{n=1}^{\omega}=\sigma_{\mathrm{p}}\left(V_{\phi}\right)=\bigcup_{i \in \Omega} \sigma_{\mathrm{p}}\left(\left(a_{i}-a_{i-1}\right) V_{\phi_{i}}\right)=\bigcup_{i \in \Omega}\left(a_{i}-a_{i-1}\right)\left\{\lambda_{i n}\right\}_{n=1}^{\omega_{i}} .
$$

By Theorem 4.8,

$$
\lim _{\varepsilon \rightarrow 0} \sum_{\left|\lambda_{i n}\right|>\varepsilon} \lambda_{i n}=1
$$

Thus

$$
\begin{aligned}
\lim _{\varepsilon \rightarrow 0} \sum_{\left|\lambda_{n}\right|>\varepsilon} \lambda_{n} & =\sum_{i \in \Omega}\left(a_{i}-a_{i-1}\right) \lim _{\varepsilon \rightarrow 0} \sum_{\left|\lambda_{i n}\right|>\varepsilon} \lambda_{i n} \\
& =\sum_{i \in \Omega}\left(a_{i}-a_{i-1}\right)=\operatorname{mes}\{x: \phi(x) \geq x, x \in[0,1]\}
\end{aligned}
$$

REMARK 5.4. It is interesting to note that the case of nonincreasing function $\phi$ can be more multifarious. In particular, if $\phi$ is a strictly decreasing 
continuous function such that $\phi(0)=1, \phi(1)=0$ and $\phi(\phi(x))=x$ then $V_{\phi}$ is a selfadjoint operator in $L_{2}[0,1]$. For example,

$$
\sigma_{\mathrm{p}}\left(V_{1-x}\right)=\left\{\frac{2(-1)^{n}}{(2 n+1) \pi}\right\}_{n=1}^{\infty}
$$

and

$$
\sum_{n=1}^{\infty} \frac{2(-1)^{n}}{(2 n+1) \pi}=\frac{2}{\pi} \frac{\pi}{4}=\frac{1}{2}=\operatorname{mes}\{x: 1-x \geq x\} .
$$

5.2. In this subsection we consider an operator $V_{\phi}$ defined on $L_{p}[0,1]$ $(1 \leq p<\infty)$.

Let $A_{i}$ be a bounded operator defined on Banach space $X_{i}(i=1,2)$. Recall that $A_{1}$ is said to be quasisimilar to $A_{2}$ if there exist deformations $K: X_{1} \rightarrow X_{2}$ and $L: X_{2} \rightarrow X_{1}$ (i.e. $\overline{\mathfrak{R}(K)}=X_{2}$, $\operatorname{ker} K=\{0\}, \overline{\mathfrak{R}(L)}=X_{1}$, ker $L=\{0\})$ such that $A_{1} L=L A_{2}$ and $K A_{1}=A_{2} K$. It is clear that $\sigma_{\mathrm{p}}\left(A_{1}\right)=\sigma_{\mathrm{p}}\left(A_{2}\right)$.

Proposition 5.5. Let $\phi:[0,1] \rightarrow[0,1]$ be a strictly increasing continuous function such that $\phi(0)=0$ and $\phi(1)=1$. Let $A_{1}$ denote an operator $V_{\phi}$ defined on $L_{p}[0,1](1 \leq p<\infty)$ and let $A_{2}$ denote $V_{\phi}$ defined on $L_{2}[0,1]$. Then $A_{1}$ is quasisimilar to $A_{2}$, and hence $\sigma_{\mathrm{p}}\left(A_{1}\right)=\sigma_{\mathrm{p}}\left(A_{2}\right)$.

Proof. Set $K:=V_{\phi}: L_{p}[0,1] \rightarrow L_{2}[0,1], L:=V_{\phi}: L_{2}[0,1] \rightarrow L_{p}[0,1]$. It is clear that $K$ and $L$ are deformations and $A_{1} L=L A_{2}, K A_{1}=A_{2} K$.

5.3. Now we consider the operator

$$
\left(V_{\phi, q, w} f\right)(x):=q(x) \int_{0}^{\phi(x)} f(t) w(t) d t
$$

defined on $L_{2}[0,1]$. The proof of the following theorem is similar to the proof of Theorem 3.2 .

THEOREM 5.6. Let $\phi:[0,1] \rightarrow[0,1]$ be a nondecreasing continuous function such that $\phi(x)>x$ for all $x \in(0,1)$. Let $q, w \in L_{2}[0,1]$. Then

$$
\begin{aligned}
& D_{V_{\phi, q, w}}(\lambda) \\
& =1+\sum_{n=1}^{\infty}(-1)^{n} \lambda^{n} \int_{0}^{1} \int_{\phi\left(t_{1}\right)}^{1} \ldots \int_{\phi\left(t_{n-1}\right)}^{1} q\left(t_{1}\right) w\left(t_{1}\right) \ldots q\left(t_{n}\right) w\left(t_{n}\right) d t_{n} \ldots d t_{1} .
\end{aligned}
$$

COROLlary 5.7. Under the assumptions of Theorem 5.6 suppose that $q(x) w(x)>0$ for a.a. $x \in[0,1]$. Then $\sigma_{\mathrm{p}}\left(V_{\phi, q, w}\right) \backslash\{0\}$ is a finite set if and only if $\phi(0)>0$ and $\phi(1-\varepsilon)=1$ for some $0<\varepsilon<1$.

Acknowledgments. The author wishes to thank Professor J. Zemánek for setting the problem. 


\section{References}

[1] I. Yu. Domanov, On the spectrum and eigenfunctions of the operator $(V f)(x)=$ $\int_{0}^{x^{\alpha}} f(t) d t$, in: Perspectives in Operator Theory, Banach Center Publ. 75, Inst.
Math., Polish Acad. Sci., 2007, 137-142.

[2] -, On cyclic subspaces and the unicellularity of the operator $(V f)(x)=$ $q(x) \int_{0}^{x} w(t) f(t) d t$, Ukr. Mat. Visn. 1 (2004), 172-213, 283 (in Russian); English transl.: Ukr. Math. Bull. 1 (2004), 177-219.

[3] - Spectral analysis of powers of the operator $(V f)(x)=q(x) \int_{0}^{x} w(t) f(t) d t$, Mat. Zametki 73 (2003), 444-449 (in Russian); English transl: Math. Notes 73 (2003), 408-413.

[4] I. C. Gohberg and M. G. Krĕn, Introduction to the Theory of Linear Nonselfadjoint Operators, transl. from the Russian by A. Feinstein, Transl. Math. Monogr. 18, Amer. Math. Soc., Providence, RI, 1969.

[5] - - - Theory and Applications of Volterra Operators in Hilbert Space, transl. from the Russian by A. Feinstein, Transl. Math. Monogr. 24, Amer. Math. Soc., Providence, RI, 1970.

[6] E. Goursat, A Course in Mathematical Analysis. Vol. III, Part Two: Integral Equations. Calculus of Variations, transl. by H. G. Bergmann, Dover, New York, 1964.

[7] B. Ya. Levin, Lectures on Entire Functions, transl. from the Russian by V. Tkachenko, Transl. Math. Monogr. 150, Amer. Math. Soc., Providence, RI, 1996.

[8] W. V. Lovitt, Linear Integral Equations, Moscow, 1957 (in Russian).

[9] Yu. I. Lyubich, Linear functional analysis, Current problems in mathematics. Fundamental directions, Vol. 19, 5-305, 316, Itogi Nauki i Tekhniki, Akad. Nauk SSSR, Vsesoyuz. Inst. Nauchn. i Tekhn. Inform., Moscow, 1988 (in Russian).

[10] - Composition of integration and substitution, in: Linear and Complex Analysis Problem Book, Lecture Notes in Math. 1043, Springer, Berlin, 1974, 249-250.

[11] Y. S. Tong, Quasinilpotent integral operators, Acta Math. Sinica 32 (1989), 727-735 (in Chinese).

[12] F. G. Tricomi, Integral Equations, Dover, New York, 1957.

[13] R. Whitley, The spectrum of a Volterra composition operator, Integral Equations Operator Theory 10 (1987), 146-149.

[14] P. P. Zabreı̌ko, A. I. Koshelev, M. A. Krasnosel'skiı̌, S. G. Mikhlin, L. S. Rakovshchik, and V. Ya. Stetsenko, Integral Operators, Nauka, Moscow, 1968 (in Russian).

[15] M. Zima, A certain fixed point theorem and its applications to integral-functional equations, Bull. Austral. Math. Soc. 46 (1992), 179-186.

Institute of Applied Mathematics

and Mechanics

Ukrainian National Academy of Sciences

R. Luxemburg St. 74

83114 Donetsk, Ukraine

E-mail: domanovi@yahoo.com
Mathematical Institute of the Academy of Sciences of the Czech Republic Žitná 25

CZ-115 67 Praha 1, Czech Republic

Received January 12, 2006

Revised version December 17, 2007 